\title{
Multi Class Traffic Analysis of Single and Multi-band Queuing System
}

\author{
Husnu S. Narman, Md. Shohrab Hossain and Mohammed Atiquzzaman \\ School of Computer Science, University of Oklahoma, Norman, OK 73019. \\ Email: \{husnu, shohrab, atiq\}@ou.edu
}

\begin{abstract}
To facilitate higher bandwidth for multimedia traffic, modern routers support simultaneous multi-band communication, leading to less interference, higher capacity and better reliability. However, there is lack of quantitative evaluation to judge whether multi-band is better than single band router in realistic scenarios. Our objective is to propose a scheduling algorithm for multi-band routers and compare single band and multi-band system with different allocation policies. We have used different scheduling algorithms for multi-band routers which transmit different classes of traffic through different frequency bands, thereby achieving improved performance. By comparing multi-band and single band mobile router performances, we have found out that one of them is not always better than the other although multi-band is expected to have better performance.

Index Terms-Analytical modeling, scheduling algorithm, queuing system, real-time traffic, next generation mobile routers.
\end{abstract}

\section{INTRODUCTION}

In recent years, there have been explosive growth of users accessing large multimedia files (such as, high definition audio, video, images, etc.) over the Internet. Therefore, the bandwidth demand for mobile Internet access is increasing exponentially [1]. To satisfy such a higher bandwidth requirement, today wireless routers are available commercially with simultaneous multi-band support of 2.4 and $5 \mathrm{GHz}$. The benefit of using multi-band router is less interference, higher capacity and better reliability.

Current simultaneous multi-band MRs make use of 2.4 and $5 \mathrm{GHz}$ for different types of devices in a home network. However, they do not attempt to exploit the under-utilized frequency band when other one is flooded with data. The multi-band router system is a heterogeneous multi-server system which means each server's service rate is different than the other. Hence, allocation policy, flexibility and priority of class of packets [2] in heterogeneity of a system must be taken into account since some of the traffic types (such as, real-time) have strict delay constraints [3]; some other signaling traffic (required for mobility management) [4] is crucial for maintaining Internet connectivity of the mobile users. Therefore, it is essential to propose an appropriate scheduling and queue management scheme for the multiclass traffic to ensure the maximum possible utilization of the system resources in multi-band mobile routers [5]. The aim of this work is to propose a scheduling algorithm for multi-band routers and compare single band and multi-band system with different allocation policies and find out under which circumstances single band or multi-band performs better through the use of different router service rates and buffer sizes.

There have been several research works [1], [5]-[8] reported in the literature. Verma and Lee [8] explain possible Wi-Fi architecture with multiple physical and link layers to support multiple frequency bands simultaneously. Singh et al. [1] proposed a method to assign different frequency bands to end-devices based on their distances from the access router. In [6], [7], authors proposed the use of $60 \mathrm{GHz}$ frequency band (having low range) to attain faster data transfer rate in wireless networks. However, none of these works [1], [5]-[8] propose any scheduling algorithm for multi-band system considering multi-class traffic, neither do they perform any comparison between multi and single bands.

To the best our knowledge, there has been no earlier works on scheduling and queue management for multi-band mobile routers that attempts to maximize utilization of available bands. Moreover, Hossain et al. [9] is the only previous work exists that proposed the sharing of multiple bands to transmit different classes of traffic. In [9], we have compared the current multi-band scheduling with our proposed multiband scheduling. However, it is essential to compare single band with our proposed scheduling for different allocations policies. This is a novel work that aims at attaining maximum possible band utilization with different allocation policies while comparing the performance of band sharing of multiband and single band routers. The objective of this work is to determine whether such a multi-band router architecture performs better than single band architecture. The contributions of this work are: (i) proposing a band-sharing router architecture and a novel scheduling algorithm that aims at improved utilization of the system by using different allocation policies, (ii) comparing the performance of multi-band router with single band router with realistic simulations, and (iii) analyzing the results to make recommendations for choosing single or multi-band architecture and allocation policies based on traffic conditions, and their priority.

Results show the packet drop rate and throughput are significantly improved in proposed band-sharing architecture of the mobile router. Moreover, multi-band router can suffer low band utilization under light traffic.

The rest of the paper is organized as follows. In Section II, 


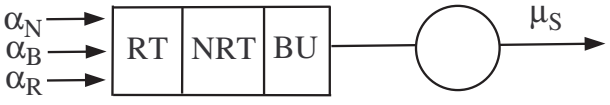

Fig. 1. Single band mobile router architecture.

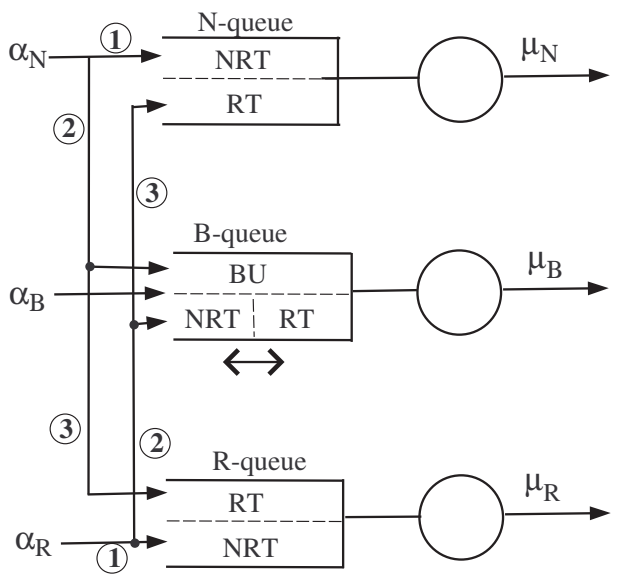

Fig. 2. Proposed architecture of a simultaneous multi-band mobile router.

we explain the typical architecture of single band mobile routers, followed by the proposed multi-band architecture in Section III. Section IV presents analysis of model to derive different performance metrics of the proposed architecture. In Section V, we present the simulation results showing the performance of queue and class differences for single and multi-band architectures. Finally, Section VI has the concluding remarks.

\section{Single BAND ROUTER ARCHITECTURE}

Traditional single band MRs use only one frequency band for all types of traffic. Fig. 1 shows the architecture of a single band MR with arrival rates of different class of traffic: signaling traffic or Binding Update (BU), non-real time (NRT), and real-time (RT) traffic with $\alpha_{B}, \alpha_{N}$ and $\alpha_{R}$ arrival rates. All the traffic are queued and served by the single server with rate $\left(\mu_{S}\right)$ based on the priority level of each class. Generally, BU packets are given the highest priority, then NRT and RT are served [10], [11]. Problem of priority scheduling (of different traffic classes) in single band architecture is that one type of packet may be served continuously while others may suffer starvation. To prevent such starvation, a threshold is used for each class. However, identifying an optimum threshold is another problem. In our model, absolutely non-preemptive priority is used for each class.

\section{Proposed MUlti-BAND ROUter ARChitecture}

Commercial mobile routers available today makes use of two different bands $(2.4 \mathrm{GHz}$ and $5 \mathrm{GHz})$ simultaneously for different types of devices in a home network. However, they do not allow sharing of bands among the traffic classes. In this section, we explain our proposed architecture of multiband MRs that promotes sharing of bands to maximize system utilization. We have considered three different queues (shown in Fig. 2), each of which corresponds to a frequency band of a simultaneous tri-band Mobile Router. We name them as Bqueue, $\mathrm{N}$-queue and $\mathrm{R}$-queue as they are designated to carry BU, NRT and RT traffic, respectively in usual case where the traffic arrival is much less than the capacity of the queue. The three queues and their corresponding arrival rates and service rates are shown in Fig. 2.

In our proposed architecture, traffic of one class can flow through other queues provided the other queues have empty slots, thereby ensuring better utilization of buffer spaces available. For example, if the B-queue has some empty spaces available and a bursty RT traffic comes in, the overflowed RT traffic can be queued in the B-queue and subsequently served (or sent) through the B-server (transmitter).

\section{A. Time and space priority}

The time and space priority for the three queues of the proposed architecture are explained in Figs. 2. For B-queue, BU packets have the highest priority; RT and NRT packets have dynamic priority based on arrival rates (see Eqns. (1) and (2)). Regarding space priority, BU packets are queued in front of B-queue and if there are empty spaces available, other types (RT and NRT) can be accommodated as shown in Fig. 2.

R-queue can have only RT and NRT packets as shown in Fig. 2. RT traffic has higher priority over NRT traffic. Therefore, R-queue can have NRT packets only if RT packets cannot fill the R-queue at any instant and there are NRT packets overflowed from the $\mathrm{N}$-queue.

Finally, if the N-queue, which is designated for NRT traffic, has empty spaces, overflowed RT traffic out of R-queue can be enqueued in N-queue (see Fig. 2).

\section{B. Allocation Policy}

We have considered the following two crucial factors to ensure improved performance of the multi-band MR: (i) The unused buffer space of one band can be used for other traffic types, thereby reducing the idle time of the system; (ii) Priorities of different traffic classes are also considered while selecting a particular type of packet over others.

Two types of allocation policies are used in the proposed architecture:

- Fastest server first (FSF),

- Least utilization first (LUF).

Queue allocation policies are explained as follows: (i) Attempts are first made to send different class of traffic through the designated frequency band; (ii) If there is overflow of RT or NRT packets from R-queue or N-queue, they are forwarded to other server based on the two principles: faster server first (computed by comparing $\mu_{B}, \mu_{N}$, and $\mu_{R}$ ) or lower utilization server (computed by $\alpha_{B} / \mu_{B}$ for B-queue, $\alpha_{N} / \mu_{N}$ for $\mathrm{N}$ queue, and $\alpha_{R} / \mu_{R}$ for R-queue); (iii) If there is no space available in the chosen queue, the packets are queued in the third queue (if there is space in it). Otherwise, packets are dropped from the system; (iv) The race between different class of traffic are resolved through the use of priority explained in 
Section IV-C; (v) Similar policy is enforced while dealing with each class of traffic.

\section{ANALYSIS}

In this section, we explain how various metrics are computed in single band and the proposed multi-band router architecture.

\section{A. Assumptions}

To make the model analytically tractable, the following assumptions have been made: (i) Packet arrival follows Poisson distribution, (ii) Type of queue discipline is FIFO with nonpreemptive priority among various traffic classes.

\section{B. Notations}

The notations used in the analysis are listed below. To simplify our notation, we use $T \in\{\mathrm{B}, \mathrm{N}, \mathrm{R}\}$ as the common notation for different traffic class types.

$N_{T} \quad$ Queue size of $T$-queue in the MR,

$\alpha_{T} \quad$ Total packet arrival rate at $T$ class of $i$-th $\mathrm{MN}$,

$\mu_{T} \quad$ Service rate at $T$-queue of $i$-th $\mathrm{MN}$,

$\sigma_{T_{B Q}} \quad$ Priority of class $T$ traffic in B-queue,

$E\left(D_{T}\right) \quad$ Average delay of class $T$ packets,

$E\left(n_{T}\right) \quad$ Average occupancy of class $T$ packets,

$P_{d T} \quad$ Drop probability of class $T$ packets,

$\gamma_{T} \quad$ Throughput of class $T$,

$E\left(D_{T Q}\right)$ Average delay of packets in $T$ queue,

$E\left(n_{T Q}\right)$ Average occupancy of packets in $T$ queue,

$P_{d T Q} \quad$ Drop probability of a packet in $T$ queue,

$\gamma_{T Q} \quad$ Throughput in $T$ queue,

$E\left(D_{T Q}^{T}\right)$ Average delay of class $T$ in $T$ queue,

$E\left(n_{T Q}^{T}\right)$ Average occupancy of class $T$ in $T$ queue,

$P_{d T Q}^{T} \quad$ Drop probability of class $T$ in $T$ queue,

$\chi_{T Q}^{T} \quad$ Total dropped packets of class $T$ in $T$ queue,

$\gamma_{T Q}^{T} \quad$ Throughput of class $T$ in $T$ queue,

$E\left(D_{\text {sys }}\right)$ Average delay of packets in system,

$E\left(n_{\text {sys }}\right)$ Average occupancy of packets in system,

$P_{d s y s} \quad$ Packet drop rate of system,

$\gamma_{\text {sys }} \quad$ Throughput of system.

\section{Priority}

Priorities of different classes are taken into account while allowing traffic into B-queue. Priority of BU packets in Bqueue is $\sigma_{B B}=1$. Priorities of other classes of traffic in $\mathrm{B}$-queue are measured as follows:

$$
\begin{aligned}
\sigma_{N_{B Q}} & =\frac{\alpha_{R}}{\alpha_{B}+\alpha_{R}+\alpha_{N}} \\
\sigma_{R_{B Q}} & =\frac{\alpha_{N}}{\alpha_{B}+\alpha_{R}+\alpha_{N}}
\end{aligned}
$$

D. Alternative way of deriving metrics using $M / M / 1 / N$ formula

We may have used $\mathrm{M} / \mathrm{M} / 1 / \mathrm{N}$ [12] formula rather than simulations to derive metrics of multi-band system by using total arrival rate of each class, total service rate and total buffer size of each band. However, the analytical formulation will not reflect the real scenario. This is because in heterogeneous multi-server system and for LUF case, packets may remain in the slower server queue, thereby increasing the packet delay and queue occupancy of the system.

Standard equations of $\mathrm{M} / \mathrm{M} / 1 / \mathrm{N}$ [12] are listed as follows;

$$
\begin{gathered}
E(n)= \begin{cases}\frac{\rho-(N+1) \rho^{N+1}+N \rho^{(N+2)}}{(1-\rho)\left(1-\rho^{N+1}\right)} & \text { if } \rho \neq 1 \\
\frac{N}{2} & \text { if } \rho=1\end{cases} \\
P_{d}= \begin{cases}\frac{\rho^{N}(1-\rho)}{1-\rho^{N+1}} & \text { if } \rho \neq 1 \\
\frac{1}{N+1} & \text { if } \rho=1\end{cases}
\end{gathered}
$$

where $\rho=\alpha / \mu$. It can be noted that all the system performance metrics (e.g., average occupancy, packet delay, drop rate, throughput) can be obtained (or approximated) only for the FSF policy under heavily loaded (server) condition. This cannot be approximated when the servers remain idle or for the LUF policy. For the FSF policy (with heavy traffic), the required parameters can be computed as follows:

$$
\begin{aligned}
& \alpha_{A l l}=\alpha_{B}+\alpha_{N}+\alpha_{R}, \mu_{A l l}=\mu_{B}+\mu_{N}+\mu_{R} \\
& N_{\text {All }}=N_{B}+N_{N}+N_{R}, \rho_{\text {All }}=\alpha_{\text {All }} / \mu_{\text {All }} .
\end{aligned}
$$

Therefore, estimated occupancy of multi-band system can be obtained using the standard equation of $M / M / 1 / N$ [12] by substituting $N=N_{A l l}, \rho=\rho_{A l l}$ into equations (3) and (4) to obtain $E\left(n_{s y s}\right)$ and $P_{d s y s}$, respectively. However, performance metrics for each class cannot be approximated in a similar way because the system can only be heavily loaded by one type packet. By using similar approach as above, it is not possible to understand which type packet has more influence on the system. To resolve that problem, our previous work [9] can be a solution for a particular case in multi-band system. On the other hand, average occupancy, delay, drop rate and throughput of each class can be measured by using approximation formulas for single band. There are some previous works [13], [14] in the literature regarding such formalations where drop rate of each class [13] and average class occupancy and delay [14] have been analytically formalized for non-preemptive priority. However, none of works has been justified by simulations. Therefore, extensive simulations have been used in this work to compare single and multi-band systems to obtain credible results. We present some alternative approximate results for the following cases in FSF allocation policy:

Case 0: BU packets are not overflowed at any time (general assumption).

Case 1: Only NRT type packets are overflowed.

Case 2: Only RT type packets are overflowed.

Case 3: Both NRT and RT types packets are overflowed. We present the analyis for only one case (Case 1). Similar methodology can be used to approximate diffenrent performance metrics based on the allocation policy.

1) Case 1: In this case, only NRT packet are overflowed and $\mu_{R}>\mu_{B}$. Hence, following approximations can be used to evaluate performances of each class.

$$
\begin{aligned}
E\left(n_{T}\right) & =E\left(n_{T Q}^{T}\right) \\
& = \begin{cases}\frac{\rho_{T}-\left(N_{T}+1\right) \rho_{T}^{N_{T}+1}+N_{T} \rho_{T}^{\left(N_{T}+2\right)}}{\left(1-\rho_{T}\right)\left(1-\rho_{T}^{N_{T}+1}\right)} & \text { if } \rho_{T} \neq 1 \\
\frac{N_{T}}{2} & \text { if } \rho_{T}=1\end{cases}
\end{aligned}
$$




$$
P_{d T}=P_{d T Q}^{T}= \begin{cases}\frac{\rho_{T}^{N_{T}}\left(1-\rho_{T}\right)}{1-\rho_{T}^{N_{T}+1}} & \text { if } \rho_{T} \neq 1 \\ \frac{1}{N_{T}+1} & \text { if } \rho_{T}=1\end{cases}
$$

Using $E\left(n_{T}\right)$ and $P_{d T}$, delay and throughput for BU and RT type packets can be obtained as follows;

$$
\begin{gathered}
E\left(D_{T}\right)=E\left(D_{T Q}^{T}\right)=\frac{E\left(n_{T}\right)}{\alpha_{T}} \\
\gamma_{T}=\gamma_{T Q}^{T}=\alpha_{T}\left(1-P_{d T}\right)
\end{gathered}
$$

where $\rho_{T}=\alpha_{T} / \mu_{T}$ and $T$ can be $B$ and $R$. However, performance evaluation for NRT type is different from BU and RT types because overflowed NRT packets are forwarded to the B-queue and R-queue. Therefore, overflowed packets should be considered while deriving performance metrics of NRT type packets.

$$
P_{d N Q}= \begin{cases}\frac{\rho_{N}^{N_{N}}\left(1-\rho_{N}\right)}{1-\rho_{N}^{N_{N}+1}} & \text { if } \rho_{N} \neq 1 \\ \frac{1}{N_{N}+1} & \text { if } \rho_{N}=1\end{cases}
$$

To find NRT packet occupancy in multi-band system, we have to know the average occupancy of NRT packets in Rqueue and B-queue. From the assumption (FSF allocation policy with $\mu_{R}>\mu_{B}$ ), it is known that overflow NRT packets are forwarded to R-queue first and then to B-queue. Therefore, average overflowed NRT packets can be measured as follow:

$$
\chi_{N Q}^{N}=\alpha_{N} P_{d N Q}
$$

To find the average occupancy of NRT type packets in Rqueue, total occupancy of R-queue is measured as follow;

$E\left(n_{R Q}\right)= \begin{cases}\frac{\rho_{R}^{\prime}-\left(N_{R}+1\right) \rho_{R}^{\prime}{ }^{N_{R}+1}+N_{R} \rho_{R}^{\prime}\left(N_{R}+2\right)}{\left(1-\rho_{R}^{\prime}\right)\left(1-\rho_{R}^{\prime} N_{R}+1\right)} & \text { if } \rho_{R}^{\prime} \neq 1 \\ \frac{N_{R}}{2} & \text { if } \rho_{R}^{\prime}=1\end{cases}$

where $\rho_{R}^{\prime}=\frac{\chi_{N Q}^{N}+\alpha_{R}}{\mu_{R}}$. Hence, average occupancy of NRT type packets in R-queue is $E\left(n_{R Q}^{N}\right)=E\left(n_{R Q}\right)-E\left(n_{R}\right)$. To find the occupancy of NRT type packets in B-queue, overflow NRT packets from R-queue should be measured by considering nonpreemptive priority since NRT packets have the second priority in R-queue. By using formula in [13], $P_{d R Q}^{N}$ can be measured. Therefore, average overflow NRT packets from R-queue can be calculated as follow:

$$
\chi_{R Q}^{N}=\alpha_{N} P_{d N Q} P_{d R Q}^{N}
$$

To find average occupancy of NRT type packets in B-queue, total occupancy of B-queue is measured as follow;

$$
E\left(n_{B Q}\right)= \begin{cases}\left.\frac{\rho_{B}^{\prime}-\left(N_{B}+1\right) \rho_{B}^{\prime} N_{B}+1}{\left(1-\rho_{B}^{\prime}\right)\left(1-\rho_{B}^{\prime}{ }_{B}^{\prime}{ }_{B}{ }_{B}+1\right.}\right) & \text { if } \rho_{B}^{\prime} \neq 1 \\ \frac{N_{B}}{2} & \text { if } \rho_{B}^{\prime}=1\end{cases}
$$

where $\rho_{B}^{\prime}=\frac{\chi_{R Q}^{N}+\alpha_{B}}{\mu_{B}}$. Hence, average occupancy of NRT type packets in B-queue is $E\left(n_{B Q}^{N}\right)=E\left(n_{B Q}\right)-E\left(n_{B}\right)$. Therefore, $E\left(n_{N}\right)=E\left(n_{N Q}\right)+E\left(n_{R Q}^{N}\right)+E\left(n_{B Q}^{N}\right) . P_{d B Q}^{N}$ can be measured in a similar manner [13]. Actually, $P_{d N}=$
$P_{d B Q}^{N}$ since only NRT packets dropped from system are the NRT packets dropped from the B-queue. By using $E\left(n_{N}\right)$ and $P_{d N}$, delay and throughput can be calculated as follows:

$$
\begin{gathered}
E\left(D_{N}\right)=\frac{E\left(n_{N}\right)}{\alpha_{N}} \\
\gamma_{N}=\alpha_{N}\left(1-P_{d N}\right)
\end{gathered}
$$

Similar approaches can be used for other cases. Similar approaches to derive performance metrics for particular case of multi-band syetems are justified in [9].

Utilization: Utilization is the percentage of time the server is busy. We have computed the band utilization through simulations by using the ratio of amount of time server was found busy to the total time of simulation in each run.

Average Queue Occupancy: We have taken average of the three queue occupancies of multi-band architecture in order to compare multi-band system with single band. Total average queue occupancy of multi-band architecture (in all queues) can be computed as follows:

$$
E\left(n_{\text {Total }}^{M B}\right)=E\left(n_{B}\right)+E\left(n_{N}\right)+E\left(n_{R}\right)
$$

Drop Probability: For multi-band system, after finding the drop rate of each packet type, we have computed the packet drop rate of the system (in simulations) as follows :

$$
P_{d(a v g)}^{M B}=\frac{\alpha_{B} P_{d B}+\alpha_{R} P_{d R}+\alpha_{N} P_{d N}}{\alpha_{B}+\alpha_{N}+\alpha_{R}}
$$

Throughput: The total throughput of multi-band MR architecture can be obtained as follows:

$$
\gamma_{A l l}^{M B}=\gamma_{B}+\gamma_{N}+\gamma_{R}
$$

Average Packet Delay: Average delay of each packet in the multi-band architecture can be obtained as follows:

$$
E\left(D_{\text {avg }}^{M B}\right)=\frac{\gamma_{B} E\left(D_{B}\right)+\gamma_{N} E\left(D_{N}\right)+\gamma_{R} E\left(D_{R}\right)}{\gamma_{A l l}^{M B}}
$$

\section{RESULTS}

We have written discrete event simulation programs in MATLAB environment by taking into account all the assumptions and scheduling policies mentioned in Sections II and III. We have followed $M / M / 1 / N$ and $M / M / 3 / N$ [12] procedures for the implementation of simulation programs. We have kept equal buffer lengths (of 50 packets) for each multi-band queue. Buffer lengths are kept small [15] similar to real routers to reduce packet delay. However, to have fair comparison with the single band architecture, total buffer length for single band is used 150 packets which is three times of multi-band buffer length. RT and NRT packets are assumed to be 512 bytes [6], [16] whereas the BU packets are assumed to be 64 bytes. The service rates of the $\mathrm{B}, \mathrm{N}$ and R-queues are kept 27, 75 and 132 packets/sec which is proportional to service rates of multiband routers [6]. Single band routers can only have one band; therefore, the highest service rate in multi-band architectue (i.e., 132 packets/sec) is used for the service rate of single band. We ran each simulation with 100000 samples for 20 trials having different traffic class arrival rates as follows: 


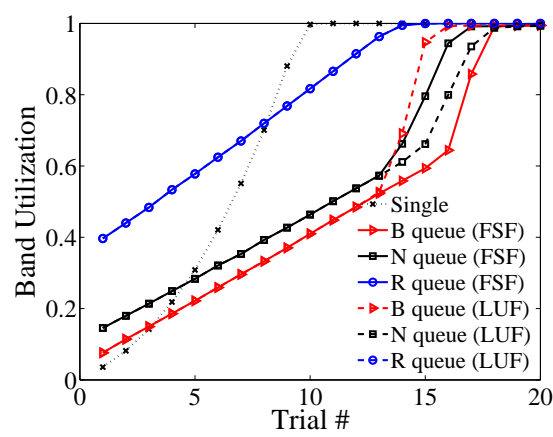

Fig. 3. Band Utilization of Single, FSF and LUF.

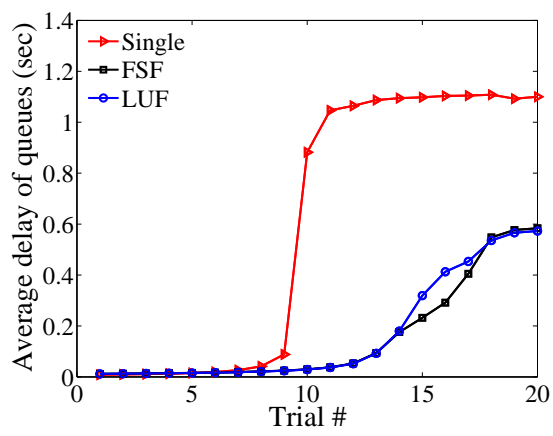

Fig. 4. Average queue delay of Single, FSF and LUF.

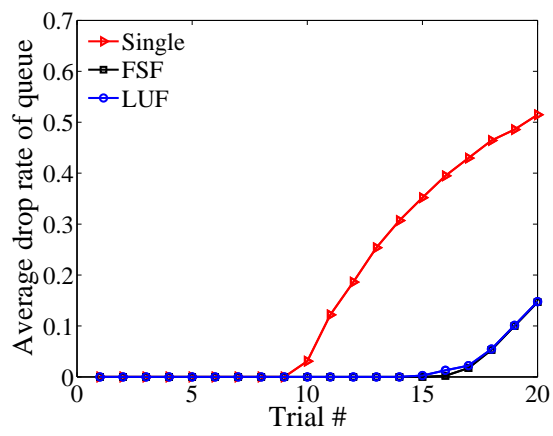

Fig. 5. Average queue drop rate of Single, FSF and LUF.
$\lambda_{B}(i)=\{\mathrm{i}\}, \lambda_{N}(i)=\{3 \mathrm{i}\}, \lambda_{R}(i)=\{10 \mathrm{i}\}$ where $\mathrm{i}=1$, $2,3, \ldots, 20$.

We have run simulations with increased arrival rates of all types of traffic to observe the impact of heavy traffic on the multi-band system. The arrival rate of B-queue and $\mathrm{N}$-queue are increased slowly in each trial whereas the RT traffic arrival rate are increased at a much higher rate. This eventually saturates the R-queue and we explain the impact of this overflow on different performance metrics of our proposed multi-band FSF and LUF allocations systems and typical single band system.

\section{A. Quеиe-wise Analysis}

We present four sets of results to show queue-wise analysis.

1) Utilization: Band utilization results for each trial are shown in Figs. 3. Single band utilization is lower than all queues of FSF and LUF under light traffic. However, under heavy traffic, multi-band utilization increases gradually but not as fast as the single band. B-queue utilization of FSF is the lowest because of the low arrival rates of BU packets and forwarding of the overflowed RT packets to N-queue first, then to B-queue. Bands utilization of LUF varies because forwarding RT class packet to other queues depends on the rate of $\alpha / \mu$. Therefore, B-queue utilization is lower than $\mathrm{N}$ queue until 15th trial, then it rises sharply. After the system reaches the maximum capacity in multi-band, utilization of bands is similar for both FSF and LUF allocations. However, it is hard to understand whether FSF band utilization is better than LUF band utilization.

2) Average queue delay and drop rate: Average queue delay and drop rate results for each trial are given in Figs. 4 and 5, respectively. Single band and multi-band delays and drop rate are significantly low while system is under light traffic. However, under heavy traffic, delay of single band sharply increases and saturates at its maximum capacity. While there is no significant differences between average delay of FSF and LUF in multi-band system, their average delay is two times better than single band.

In Fig. 5, average drop rate of multi-band for FSF and LUF is found to be lower than single band architecture because total service rate of multi-band is almost two times of that of single band. It is also interesting to see that FSF drop rate are similar to LUF drop rate.

According to queue-wise analysis results, following observations are obtained: (i) performance of multi-band architecture (for both allocation policies) is better than single band architecture under heavy traffic, (ii) multi-band systems do not use band efficiently as single band while system is under low traffic, and (iii) FSF allocation policy in multi-band architecture has the best performance.

\section{B. Class-wise Analysis}

We present average class delay and class drop rate to show class-wise analysis. Average class delays for each trial are given in Figs. 6, 7, and 8. BU and NRT class delays in single band are low. Under heavy RT traffic, RT class delay sharply increases in single band because of priority order of BU, NRT, and RT of single band. Interestingly, total service rate of multi-band is almost two times higher than service rate of single band, RT class delay of single band is at least three times higher than RT class delay of multi-band (see Fig. 6 and 7). Although LUF allocation of multi-band shows notable performance for RT traffic, FSF is found to be better than LUF (see Fig. 8).

Class drop rate for each trial are given in Figs. 9, 10, and 11. Under light traffic, all class drop rates are low for single and multi band architectures. However, under heavy NRT and RT traffic, RT drop rate in single band is almost three times higher than RT traffic of FSF and LUF in multi-band (see Figs. 9 and 10). There is no significant differences between RT traffic drop rate of FSF and LUF (see Fig. 11).

According to class-wise analysis results, following observations are obtained: (i) the highest priority class in single band can have less delay than same class in multi-band architecture, (ii) under heavy traffic, the lower priority class in single band has longer waiting time (in queue) than for multi-band architecture, and (iii) although FSF has less delay than LUF for RT class, there is no significant difference between throughput of FSF and LUF policies.

\section{CONCLUSiON}

In this paper, we have proposed a novel scheduling algorithm for multi-band mobile routers that exploits band sharing. 


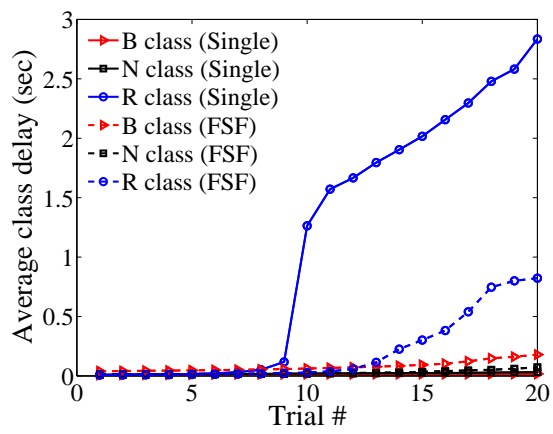

Fig. 6. Average class delay of FSF and Single.

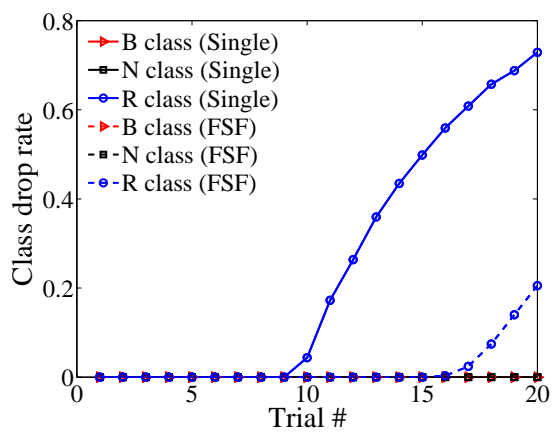

Fig. 9. Class drop rate of FSF and Single.

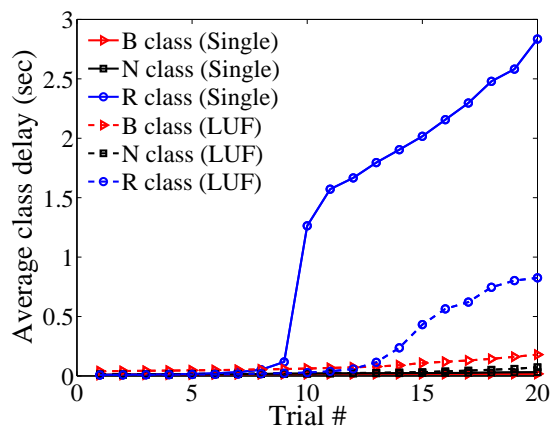

Fig. 7. Average class delay of LUF and Single.

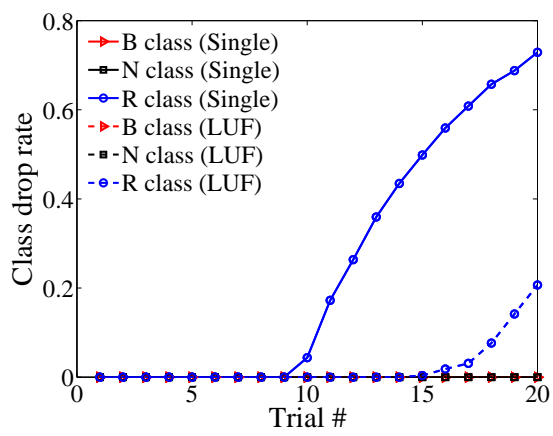

Fig. 10. Class drop rate of LUF and Single.

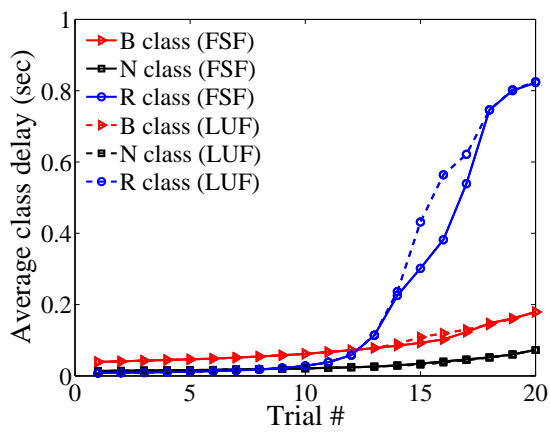

Fig. 8. Average class delay of FSF and LUF.

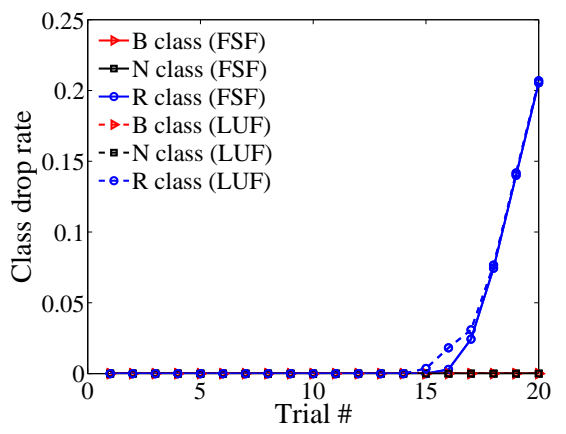

Fig. 11. Class drop rate of FSF and LUF.
Analytical formulations of the proposed multi-band system are presented through different cases for fastest server first allocation. Performance of multi-band and single band system under realistic scenarios have been compared through extensive simulations. After analyzing both the systems based on classwise and queue-wise performances, following observation are obtained: (i) single band priority system is recommended for low traffic system, (ii) multi-band system is recommended for heavy traffic systems, (iii) class priority in single band plays crucial role in class performance, and (iv) class priority and allocation policy in multi-band system has significant impact on the system performance. The results obtained in this paper can help network engineers to develop efficient routers, and also end-users to identify suitable routers to fulfill their needs.

\section{REFERENCES}

[1] H. Singh, J. Hsu, L. Verma, S. S. Lee, and C. Ngo, "Green operation of multi-band wireless LAN in $60 \mathrm{GHz}$ and $2.4 / 5 \mathrm{GHz}$," in Consumer Communications and Networking Conference (CCNC), Las Vegas, NV, Jan 9-12, 2011, pp. 787-792.

[2] T. Heath, B. Diniz, E. V. Carrera, W. M. Jr., and R. Bianchini, "Energy conservation in heterogeneous server clusters," in Principles and Practice of Parallel Programming, Chicago, IL, June 15-17, 2005, pp. $186-195$.

[3] J. Fitzpatrick, S. Murphy, M. Atiquzzaman, and J. Murphy, "Echo: A quality of service based endpoint centric handover scheme for voip," in IEEE Wireless Communications and Networking Conference, Las Vegas, NV, Mar 31- Apr 3, 2008, pp. 2777-2782.

[4] M. Hossain and M. Atiquzzaman, "Cost analysis of mobility protocols," Telecommunication Systems, vol. 52, no. 4, pp. 2271-2285, 2013.

[5] I. F. Akyildiz, D. M. Gutierrez-Estevez, and E. C. Reyes, "The evolution to 4G cellular systems: LTE-Advanced," Physical Communication, vol. 3, pp. 217-244, Mar 2010 .
[6] E. Perahia, C. Cordeiro, M. Park, and L. L. Yang, "IEEE 802.11ad: Defining the next generation multi-Gbps Wi-Fi," in 7th IEEE Consumer Communications and Networking Conference (CCNC), Las Vegas, NV, Jan 9-12, 2010.

[7] S. Singh, R. Mudumbai, and U. Madhow, "Distributed coordination with deaf neighbors: Efficient medium access for $60 \mathrm{GHz}$ mesh networks," in IEEE INFOCOM, San Diego, CA, Mar 14-19, 2010.

[8] L. Verma and S. S. Lee, "Multi-band Wi-Fi systems: A new direction in personal and community connectivity," in IEEE International Conference on Consumer Electronics (ICCE), Las Vegas, NV, Jan 9-12, 2011, pp. 665-666.

[9] M. S. Hossain, H. Narman, and M. Atiquzzaman, "A novel scheduling and queue management scheme for multi-band mobile routers," in IEEE International Conference on Communications (ICC), Budapest, Hungary, June 9-13, 2013, pp. 3787 - 3791.

[10] M. S. Hossain, M. Atiquzzaman, and W. Ivancic, "Scheduling and queue management for multi-class traffic in access router of mobility protocol," in IEEE HPCC, Melbourne, Australia, Sep 1-3, 2010, pp. 653-658.

[11] N. Nasser, L. Karim, and T. Taleb, "Dynamic multilevel priority packet scheduling scheme for wireless sensor network," IEEE Transactions on Wireless Communications, vol. 12, no. 4, pp. 1536-1276, Feb 2013.

[12] D. Gross, J. Shortle, J. Thompson, and C. M. Harris, Fundamentals of Queueing Theory. Wiley-Interscience, Aug 2008.

[13] K. E. Avrachenkov, N. O. Vilchevsky, and G. L. Shevlyakov, "Priority queueing with finite buffer size and randomized push-out mechanism," Performance Evaluation, vol. 61, Oct 2005.

[14] V. Zaborovsky, O. Zayats, and V. Mulukha, "Priority queueing with finite buffer size and randomized push-out mechanism," in International Conference on Networking, Menuires, Apr 11-16, 2010, pp. 316-320.

[15] G. Appenzeller, I. Keslassy, and N. McKeown, "Sizing router buffers," Computer Communication Review, vol. 34, pp. 281-292, Oct 2004.

[16] G. Ahn, A. T. Campbell, A. Veres, and L. Sun, "Supporting service differentiation for real-time and best-effort traffic in stateless wireless ad hoc networks (SWAN)," IEEE Transactions on Mobile Computing, vol. 1, pp. 192-207, Sept 2002. 